\title{
Microscopic neoplastic thrombosis in localised nephroblastoma: Does it influence outcome?
}

\author{
Maria Antonietta De Ioris ${ }^{a, *}$, Ivan Aloi ${ }^{b}$, Francesca Diomedi Camassei ${ }^{c}$, Lucilla Ravà ${ }^{d}$, \\ Renata Boldrini ${ }^{c}$, Luigi De Sio ${ }^{a}$, Clementina De Laurentis ${ }^{a}$, Aurora Castellano ${ }^{a}$, \\ Alessandro Crocoli ${ }^{b}$, Franco Locatelli ${ }^{a}$, Alessandro Jenkner ${ }^{a, e}$, Alessandro Inserra ${ }^{a, e}$ \\ a Paediatric Haematology/Oncology Department, Ospedale Pediatrico Bambino Gesù-IRCCS, Rome, Italy \\ ${ }^{\mathrm{b}}$ Paediatric Surgery Department, Ospedale Pediatrico Bambino Gesù-IRCCS, Rome, Italy \\ c Patology Department, Ospedale Pediatrico Bambino Gesù-IRCCS, Rome, Italy \\ d Epidemiology Unit, Ospedale Pediatrico Bambino Gesù-IRCCS, Rome, Italy
}

\section{A R T I C L E I N F O}

\section{Article history:}

Received 6 July 2010

Received in revised form 5 October

2010

Accepted 27 October 2010

Available online 2 December 2010

\section{Keywords:}

Nephroblastoma

Risk factors

Thrombosis

Outcome

\begin{abstract}
A B S T R A C T
Introduction: Microscopic neoplastic thrombosis (MNT) is reported to occur frequently in Wilms tumour (WT). The aim of this study is to determine whether MNT influences prognosis in localised WT.

Patients and methods: Records and slides of 80 consecutive, unselected, localised WT patients were retrospectively reviewed. All patients received chemotherapy before surgery according to SIOP Protocol. The median follow-up was 9 years (range 0.5-25.8). The KaplanMeier method and the Cox proportional hazard model were applied.

Results: MNT was present in 14 (18\%) cases. Out of 14 patients with MNT, 6 presented macroscopic thrombosis and 5 had either blastemal predominance or anaplastic histology. The 5 -year overall survival (OS) and progression-free survival (PFS) for the whole population were $95 \%$ (95\% confidence interval, CI, 87-98\%) and 91\% (95\% CI 82-96\%), respectively. The 5-year OS and PFS for MNT positive patients were $92 \%$ (95\% CI 57-99\%) and 77\% (95\% CI 44-92\%), while the 5-year OS and PFS for MNT negative patients were $96 \%$ (95\% CI 87-99\%) and $94 \%$ (95\% CI 85-98\%), respectively; the difference was statistically significant $(p<0.05)$ for PFS. In multivariate analysis, only the presence of anaplasia retained significance with a hazard ratio $(\mathrm{HR})$ of 14.8 and $12.9(p<0.05)$ for recurrence and death, respectively.

Conclusion: These data suggest that the presence of MNT increases the risk of recurrence. MNT is associated with well-known prognostic factors, such as macroscopic thrombosis (possibly representing regression of macroscopic involvement) and anaplasia. Further prospective studies are needed to clarify the role of MNT as independent prognostic factor.
\end{abstract}

(c) 2010 Elsevier Ltd. All rights reserved.

\section{Introduction}

Wilms tumour (WT) is the most common paediatric malignant renal tumour and also the most common cause of abdominal mass in infants and young children. ${ }^{1}$ Its incidence is 8.1 cases per million per year in Caucasian children less than 15 years of age in North America, with an incidence rate approximately three times higher for blacks. ${ }^{1,2}$

\footnotetext{
* Corresponding author: Address: Department of Paediatric Oncology-Hematology, Ospedale Pediatrico Bambino Gesù IRCCS, Piazza S. Onofrio 4, 00165 Roma, Italy. Tel.: +39 06 68592574; fax: +39 0668592242.

${ }^{e}$ These authors are equally contributed to the work. 0959-8049/\$ - see front matter (c) 2010 Elsevier Ltd. All rights reserved. doi:10.1016/j.ejca.2010.10.028
} 
Based on the currently available treatment protocols, the overall survival rate has greatly improved over the years approaching $90 \%$ and WT has become the paradigm for successful cancer treatment. ${ }^{3}$

The goal of WT trials both in Europe and in the USA has been to adapt therapy to the expected risk, in order to maximize survival while minimizing acute and late toxicities. ${ }^{4}$ Prognostic factors in WT are quite straightforward and largely accepted: the presence of metastasis, stage, histology and, in particular, anaplasia has traditionally been established as reliable predictors of survival. ${ }^{5-8}$

Renal vein and intracaval thrombosis are frequently encountered in WT and are taken into account for the purpose of staging by the current pathology guidelines., ${ }^{9,10}$ Microscopic thrombosis within intratumoural, intracapsular and intrarenal vessels is often reported by pathologists; however, there are only available data about its influence on the outcome in patients who received immediate nephrectomy. ${ }^{11-13}$

In a WT series including metastatic and bilateral disease, our group has preliminarily reported a worse prognosis of patients with microscopic neoplastic thrombosis (MNT). ${ }^{14}$

The aim of this study was to determine if MNT represents a prognostic factor in localised WT patients who had received chemotherapy before nephrectomy.

\section{Patient and methods}

\subsection{Patients}

The medical records of all consecutive WT patients diagnosed and treated at IRCCS Ospedale Pediatrico Bambino Gesù (OPBG), Rome, from January 1981 through December 2007 were retrospectively reviewed. Data regarding sex, age, tumour localisation (site and laterality) at diagnosis, stage, presence of metastasis at diagnosis, treatment, details of surgery, histology, macroscopic thrombosis and outcome were collected. The occurrence of relapse and death from any cause was registered. Patients were treated according to the SIOP (International Society of Pediatric Oncology) protocol currently in use at the time of WT diagnosis.

\subsection{Histology}

For the purpose of this study stage and histology risk group were all codified according to the most recent SIOP guidelines ${ }^{9,10}$ in order to allow cross comparison; in particular prior stage $\mathrm{II}^{+}$(with positive lymph nodes) was considered throughout as stage III. Histological sub-types were classified according to the revised SIOP working classification (2001) as low risk, intermediate risk and high risk tumours. A panel of SIOP pathologists had also centrally reviewed histology and stage at the time of diagnosis for all patients included in this study.

Microscopic thrombosis was defined as the presence of neoplastic tissue within lymphatic/venous vessels of the tumour capsule, the tumour itself and/or the surrounding renal parenchyma. When necessary for endothelium identification, antiCD34 immunostaining was performed, according to standard protocols. All cases diagnosed before 2001 were retrospectively reviewed by one of the authors (FDC) for the assessment of microscopic thrombosis. After 2001, microscopic thrombosis had been prospectively assessed in all patients.

\subsection{Statistical methods}

Overall survival (OS) was defined as the time interval between the date of diagnosis and the date of death from any cause or the date of last follow-up. Progression-free survival (PFS) was defined as the time interval between the date of diagnosis and the date of first relapse or the date of last follow-up. The Kaplan-Meier method was used for the estimation of survival curves, ${ }^{15}$ while the log-rank test was used to compare differences between groups.

Multivariate analyses were performed using Cox proportional hazards regression model for PFS and OS. Variables

Table 1 - Patients characteristics $($ No $=80)$. Histology risk group and stage were defined according to SIOP 2001 protocol.

\begin{tabular}{|c|c|c|c|c|}
\hline & & & No & $\%$ \\
\hline Age & $\begin{array}{l}\text { Mean } \pm \text { SD (months) } \\
\text { Range (months) }\end{array}$ & $\begin{array}{l}39 \pm 28 \\
3-163\end{array}$ & & \\
\hline Sex & $\begin{array}{l}\text { Male } \\
\text { Female }\end{array}$ & & $\begin{array}{l}42 \\
38\end{array}$ & $\begin{array}{l}52 \\
48\end{array}$ \\
\hline Histology & $\begin{array}{l}\text { Low risk } \\
\text { Intermediate risk } \\
\text { High risk }\end{array}$ & & $\begin{array}{r}4 \\
61 \\
15\end{array}$ & $\begin{array}{r}5 \\
76 \\
19\end{array}$ \\
\hline Anaplasia & $\begin{array}{l}\text { Focal/diffuse } \\
\text { Absent }\end{array}$ & & $\begin{array}{r}8 \\
72\end{array}$ & $\begin{array}{l}10 \\
90\end{array}$ \\
\hline MNT & $\begin{array}{l}\text { Yes } \\
\text { No }\end{array}$ & & $\begin{array}{l}14 \\
66\end{array}$ & $\begin{array}{l}18 \\
82\end{array}$ \\
\hline Macroscopic thrombosis & $\begin{array}{l}\text { Yes } \\
\text { No }\end{array}$ & & $\begin{array}{l}16 \\
64\end{array}$ & $\begin{array}{l}20 \\
80\end{array}$ \\
\hline Stage & $\begin{array}{l}\text { I } \\
\text { II } \\
\text { III }\end{array}$ & & $\begin{array}{l}48 \\
12 \\
20\end{array}$ & $\begin{array}{l}60 \\
15 \\
25\end{array}$ \\
\hline
\end{tabular}


Table 2 - Characteristics of the 14 patients with microscopic neoplastic thrombosis (MNT). Pt, patient; IVC, inferior vena cava; DOD, died of disease; NED, non evidence of disease.

\begin{tabular}{|c|c|c|c|c|c|c|c|}
\hline Pt & Sex & Age (months) & Histology & Stage & Macroscopic Thrombosis & Relapse & Outcome \\
\hline 1 & M & 40 & Mixed & 1 & No & Yes, lung & DOD, 34 \\
\hline 2 & $\mathrm{~F}$ & 26 & Regressive & 3 & Yes, IVC & & NED, 49+ \\
\hline 3 & M & 41 & Necrotic & 1 & No & & NED, 81+ \\
\hline 4 & $\mathrm{~F}$ & 52 & Blastemal & 3 & Yes, IVC & & NED, 99+ \\
\hline 5 & $M$ & 78 & Anaplastic & 2 & No & Yes, lung & DOD, 76 \\
\hline 6 & M & 32 & Mixed & 3 & Yes, IVC & & NED, $82+$ \\
\hline 7 & M & 64 & Anaplastic & 3 & No & & NED, $103+$ \\
\hline 8 & M & 3 & Epithelial & 3 & Yes, IVC & Yes, abdomen & DOD, 143 \\
\hline 9 & $\mathrm{~F}$ & 28 & Blastemal & 1 & No & & NED, $192+$ \\
\hline 10 & M & 87 & Mixed & 3 & Yes, IVC & & NED, $40+$ \\
\hline 11 & $\mathrm{~F}$ & 13 & Blastemal & 3 & Yes, Renal Vein & & NED, 26+ \\
\hline 12 & $\mathrm{M}$ & 24 & Mixed & 3 & Yes, IVC & & NED, 63+ \\
\hline 13 & $\mathrm{~F}$ & 53 & Mixed & 3 & Yes, IVC & & NED, 61+ \\
\hline 14 & $M$ & 43 & Mixed & 1 & No & & NED, 90+ \\
\hline
\end{tabular}

that reached a $p$-value of 0.20 after univariate analysis were included in the initial model and variables were eliminated one at a time in a stepwise fashion to only keep variables that reached a $p$-value of 0.05 or less into the final model. All $p$-values were 2-sided, with a type I error rate fixed at 0.05. Variables considered in risk factor analysis for PFS and OS were age ( $>2$ years or $<2$ years), stage, presence of diffuse or focal anaplasia, histology risk group, presence of macroscopic thrombosis and MNT. Analyses were performed using the Stata 9.0 statistical software package (StatCorp LP, Texas, USA).

\section{Results}

During a 27-year period, 147 consecutive WT patients were diagnosed and treated at OPBG: only patients who received pre-operative chemotherapy and presented localised disease were included in this study. The present analysis therefore concerns 80 patients. Patient characteristics, histology and stage according to SIOP are summarised in Table 1.

Patients were treated according to SIOP protocols (enrolled in SIOP-6 between 1981 and 1987, in SIOP-9 from 1987 to 1993,

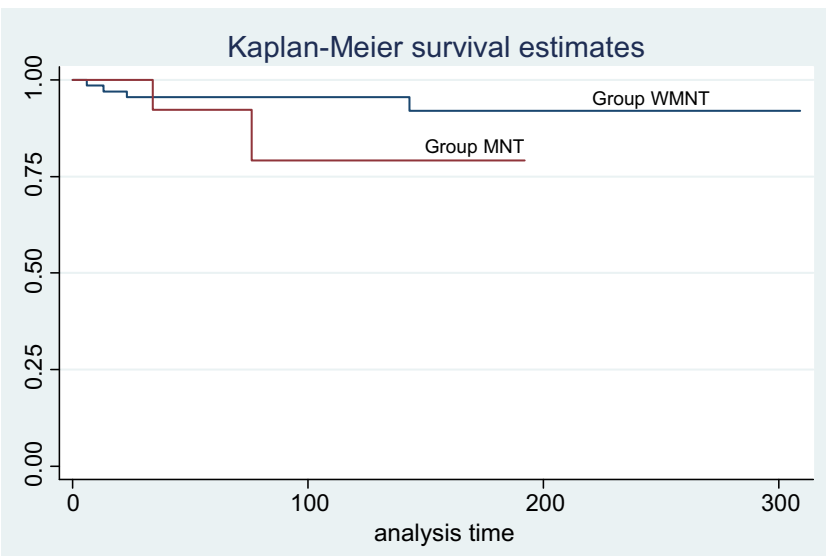

Fig. 1 - Kaplan-Meier estimates of overall survival of patients with microscopic neoplastic thrombosis (Group MNT) and of patients without microscopic thrombosis (Group WMNT). in SIOP 93-01 from 1993 to 2001 and in SIOP 2001 from 2002 onwards).

MNT was present in 14/80 (18\%) patients. Table 2 summarises the characteristics of these patients with microscopic thrombosis. Out of the 14 patients with MNT, 8 presented macroscopic thrombosis, while a blastemal subtype was observed in 3 patients and anaplasia in two. All patients with MNT but without macroscopic thrombosis were diagnosed before 1999.

The median follow-up of the entire cohort was 9 years (range 0.5-25.8 years). Of 80 evaluable patients, 6 (7.3\%) died; 5 of relapsed/resistant disease and the last one of leukaemia occurring as second neoplasia. Relapse occurred in 7 (8.7\%) patients after a median time from diagnosis of 16 months (range 6-28 months). All relapses were metastatic.

The 5-year Kaplan-Meier estimates of OS and PFS for MNT positive patients were $92 \%$ (95\% confidence interval(CI) 5799\%) and 77\% (95\% CI 44-92\%), respectively, while the 5-year OS and PFS for MNT negative patients were 96\% (95\% CI 8799\%) and 94\% (95\% CI 85-98\%), respectively (Figs. 1 and 2). The difference in OS and PFS between the two patient groups was statistically significant only for PFS $(p<0.05)$.

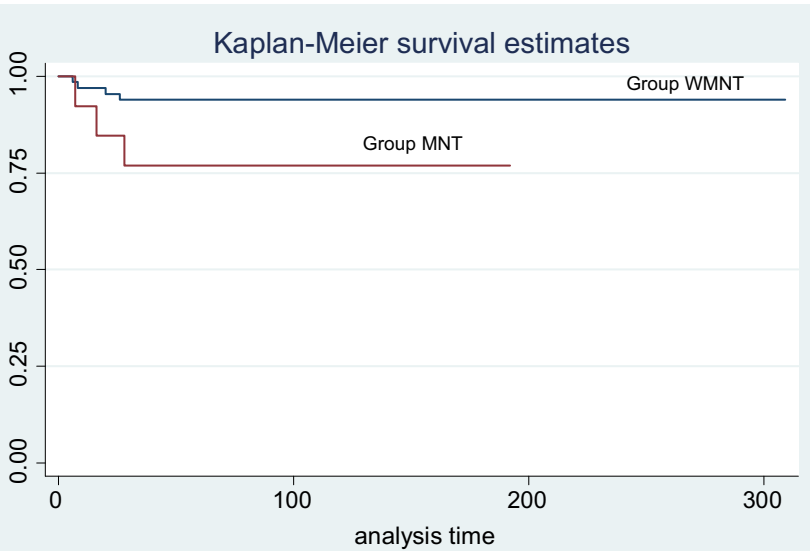

Fig. 2 - Kaplan-Meier estimates of progression-free survival of patients with microscopic neoplastic thrombosis (Group MNT) and patients without microscopic thrombosis (Group WMNT). 
Table 3 - Univariate analysis (evaluable pts 80/80). Pts patients; CI confidence interval. $\mathbf{p}<\mathbf{0 . 0 5}$.

\begin{tabular}{|c|c|c|c|c|c|c|c|c|}
\hline & & Pts & PFS 5 year (\%) & $95 \% \mathrm{CI}$ & $\begin{array}{c}\text { Univariate } \\
\text { analysis } p \text { value }\end{array}$ & OS 5 year (\%) & $95 \%$ CI & $\begin{array}{c}\text { Univariate } \\
\text { analysis } p \text { value }\end{array}$ \\
\hline \multirow[t]{2}{*}{ Age } & $>2$ year & 55 & 92 & $81-97$ & & 94 & 83-98 & \\
\hline & $<2$ year & 25 & 89 & $69-96$ & 0.634 & 96 & $76-99$ & 0.702 \\
\hline \multirow[t]{3}{*}{ Histology } & Low risk & 4 & 92 & 54-99 & & 92 & 54-99 & \\
\hline & Intermediate risk & 61 & 94 & 83-98 & & 96 & 85-99 & \\
\hline & High risk & 15 & 80 & $50-93$ & 0.223 & 93 & $61-99$ & 0.777 \\
\hline \multirow[t]{2}{*}{ Anaplasia } & Focal/diffuse & 8 & 63 & $23-86$ & & 75 & $31-93$ & \\
\hline & Absent & 72 & 94 & $86-98$ & 0.002 & 97 & 89-99 & 0.005 \\
\hline \multirow[t]{2}{*}{ MNT } & Yes & 14 & 77 & 44-92 & & 92 & $57-99$ & \\
\hline & No & 66 & 94 & $85-98$ & 0.046 & 96 & 87-99 & 0.658 \\
\hline \multirow{2}{*}{$\begin{array}{l}\text { Macroscopic } \\
\text { thrombosis }\end{array}$} & Yes & 16 & 81 & $52-94$ & & 95 & $86-98$ & \\
\hline & No & 64 & 94 & 84-98 & 0.106 & 93 & $63-99$ & 0.786 \\
\hline \multirow[t]{3}{*}{ Stage } & I & 48 & 96 & 84-99 & & 95 & 84-99 & \\
\hline & II & 12 & 92 & 54-99 & & 100 & - & \\
\hline & III & 20 & 80 & $55-92$ & 0.111 & 90 & $66-97$ & 0.415 \\
\hline
\end{tabular}

In univariate analysis only the presence of MNT and that of focal/diffuse anaplasia were statistically significant prognostic factors for recurrence $(p<0.05$, see also Table 3 for details of univariate analysis). The probability of OS was influenced only by the presence of focal/diffuse anaplasia $(p<0.01)$. The 5-year OS and PFS for patients with diffuse/focal anaplasia were $75 \%$ (95\% CI 31-93\%) and 63\% (95\% CI 23-86\%), while the 5-year OS and PFS for patients without anaplasia were 97\% (95\% CI 89-99\%) and 94\% (95\% CI 86-98\%), respectively. The difference in OS and PFS between the two patient groups was statistically significant $(p<0.05)$.

In multivariate analysis, only the presence of focal/diffuse anaplasia was prognostically significant $(p<0.05)$ with an hazard ratio (HR) of 12.9 for survival and 14.8 for recurrence $(p<0.05)$ (see Table 4). No other evaluated variables were associated with a statistically significant hazard ratio (see also Table 4 for details of multivariate analysis).

\section{Discussion}

WT is the most frequent malignant renal tumour in children. During the last three decades, multicentre trials have been carried out both in Europe and in the USA by SIOP and the National Wilms Tumour Study Group (NWTSG); the goal of all recent WT trials has been to increase survival, while reducing the morbidity associated with treatment. A risk-adapted approach is the current mainstay of treatment; stage $e^{5,6,8}$ and histology, especially the presence of anaplasia, ${ }^{7,8,16}$ remain the most important prognostic factors. Current research is seeking novel prognostic factors allowing to modulate treatment and improve risk stratification. Several biological features have been recently studied, such as the loss of heterozygosis (LOH) of chromosomes $1 \mathrm{p}$ and $16 \mathrm{q},{ }^{17}$ a high telomerase RNA expression level ${ }^{18}$ or the apoptosis factor expression, ${ }^{19}$ and the multidrug resistance P-glycoprotein expression in tumour cells and intratumoural capillary endothelial cells. ${ }^{20}$

The idea of using MNT as a prognostic factor was investigated by the NWTSG several years ago in patients who were given, according to the NWTSG strategy accepted at that time, primary nephrectomy. Breslow and colleagues evaluated prognostic factors in stage IV WT, documenting that these patients were more likely to have intrarenal thrombus as compared to patients with less advanced stages. ${ }^{11}$ Weeks and

Table 4 - Multivariate Analysis (evaluable pts 80/80). Pts patients; CI confidence interval; HR hazard ratio. $p<0.05$.

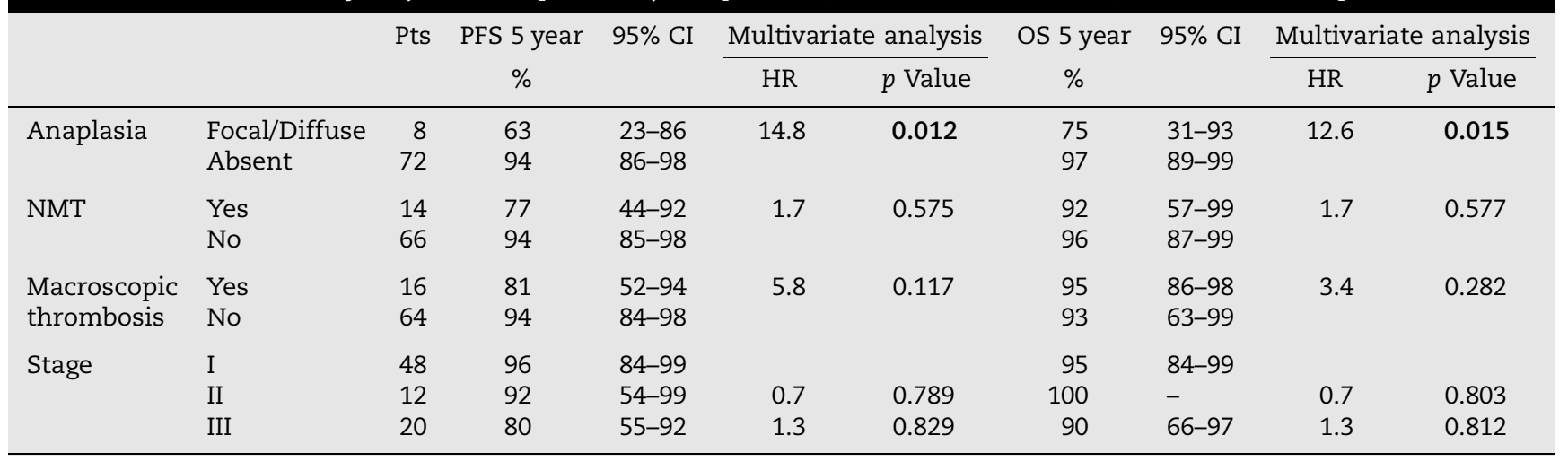


colleagues evaluated the prognostic significance of intrarenal tumour thrombus, renal sinus invasion, invasion of the tumour capsule, and the presence of an inflammatory pseudocapsule in patients with stage I WT entered in study NWTS-3: the presence of all these factors was predictive of relapse in univariate analysis, but intrarenal neoplastic thrombus was not confirmed to be a prognostic factor in multivariate analysis. ${ }^{12}$ Finally, Breslow and colleagues demonstrated invasion of intrarenal vasculature not to be a prognostic factor in non-metastatic, favourable-histology Wilms tumours. ${ }^{13}$

This retrospective study aimed at investigating a possible prognostic role of microscopic thrombosis in patients with localised WT who had received pre-operative chemotherapy, according to SIOP strategy. At the 2006 SIOP annual meeting, in a preliminary analysis, our group reported a worse prognosis in patients who presented MNT; in that series, all patients affected by histologically confirmed WT were included, independently from the choice of pre-operative chemotherapy or the presence of bilateral/metastatic disease. ${ }^{14}$ In the present cohort, patients with metastatic and bilateral WT were excluded, considering their different biology and outcome. In order to further strengthen the analysis, the slides of all patients were reviewed to confirm diagnosis and to perform restaging according to current SIOP histology guidelines for all patients diagnosed before 2001. Moreover, evaluation of MNT was performed in all cases by the same pathologist.

MNT, found in 14/80 (18\%) WT, seems to adversely affect the probability of recurrence, with a 5 -year PFS of $77 \%$ in MNT positive patients versus $94 \%$ in MNT negative patients. The difference in terms of 5 -year PFS between the groups was statistically significant $(p<0.05)$, while the minor difference in 5-year OS (92\% versus $96 \%$ ) was no longer significant.

In multivariate analysis, the prognostic value of MNT was overcome by that of anaplasia which, not surprisingly, was confirmed to be the strongest prognostic factor for disease relapse, the HR for recurrence in multivariate analysis being 14.8. The presence of anaplasia was found in $8 / 80$ patients (10\%) and influences patients' outcome both in terms of survival and risk of recurrence, while macroscopic thrombosis, found in $16 / 80$ patients (20\%), had no significant impact on outcome in our series in a univariate analysis. The 5-year OS and PFS for patients with diffuse/focal anaplasia were $75 \%$ and $63 \%$, while the 5 -year OS and PFS for patients without anaplasia were $97 \%$ and $94 \%$, respectively $(p<0.01)$. There was a significantly increased risk of death associated with the presence of anaplasia, HR for death being 12.8.

While MNT represents an easily assessed feature, it failed to identify a group of patients at increased risk of death after pre-operative chemotherapy and nephrectomy.

MNT patients were more likely to have an aggressive histology of both the blastemal or anaplastic sub-types and MNT was closely associated with macroscopic thrombosis in almost a half of the cases. We cannot exclude that the worse outcome of the MNT subgroup, in term of PFS, be probably due to these characteristics more than the presence of isolated MNT. Indeed, considering the concordance of MNT and macroscopic thrombosis found in the last decade, it is reasonable to speculate that MNT be considered as a "surrogate" and a "residual" of macroscopic thrombosis. It can be hypothesised that in our patients MNT was always associated with the presence of macroscopic thrombosis and, as that, it had regressed after chemotherapy. Moreover, as all our patients were given chemotherapy, the presence of MNT could allow to identify patients with more aggressive and/or less chemo-sensitive tumour.

One limitation of our study is that it is a retrospective analysis carried out on a population diagnosed over a long time span and consequently including patients treated according to marginally modified protocols (mainly differing in terms of reduced intensity and duration over time) and staged with different imaging tools. Indeed, over this time period, there has been a significant change in the capacity to identify vascular involvement by ultrasound and/or CT scans. A further, hopefully more definitive analysis on a larger number of patients with localised WT, homogeneously receiving the same diagnostic/therapeutic approach, such as those accrued in the most recent SIOP 2001 study, could provide a firm answer to the question whether MNT does or does not have a prognostic value, independent from its association with other variable predicting a poor outcome, such as an aggressive histology or/and macroscopic thrombosis.

\section{Grant support}

This study was partially supported by Italian Ministry of Health (MADI and IA) and "Girasole Onlus" (MADI).

\section{Conflict of interest statement}

None declared.

\section{Acknowledgements}

The authors would like to thank J. De Kraker for the useful and friendly suggestions to the work. No writing assistance was utilised in the production of this manuscript.

\section{R E F E R E N C E S}

1. Gurney JG, Severson RK, Davis S, et al. Incidence of cancer in children in the United States. Sex, race and 1 year age specific rates by histological type. Cancer 1995;75:2186-95.

2. Stiller CA, Parkin DM. International variations in the incidence of childhood renal tumour. $\mathrm{Br} J$ Cancer 1990;62:1026-30.

3. D'Angio GJ. The National Wilms Tumor Study: a 40 year perspective. Lifetime Data Anal 2007;13:463-70.

4. Mitchell C, Pritchard-Jones K, Shannon R, et al. Immediate nephrectomy versus preoperative chemotherapy in the management of non-metastatic Wilms' tumour: results of a randomised trial (UKW3) by the UK Children's Cancer Study Group. Eur J Cancer 2006;42:2554-62.

5. Tournade MF, Com-Nougué C, Voûte PA, et al. Results of the Sixth International Society of Pediatric Oncology Wilms' Tumor Trial and Study: a risk-adapted therapeutic approach in Wilms' tumor. J Clin Oncol 1993;11:1014-23.

6. Kaste SC, Dome JS, Babyn PS, et al. Wilms tumour: prognostic factors, staging, therapy and late effects. Pediatr Radiol 2008;38:2-17. 
7. Dome JS, Cotton CA, Perlman EJ, et al. Treatment of anaplastic histology Wilms' tumor: results from the fifth National Wilms' Tumor Study. J Clin Oncol 2006;24:2352-8.

8. Weirich A, Ludwing R, Graf N, et al. Survival in nephroblastoma treated according to the trial and study SIOP9/GPOH with respect to relapse and morbility. Ann Oncol 2004;15:808-20.

9. Vujanić GM, Sandstedt B, Harms D, et al. Revised International Society of Paediatric Oncology (SIOP) working classification of renal tumors of childhood. Med Pediatr Oncol 2002;38:79-82.

10. Delemarre JF, Sandstedt B, Harms D, et al. The new SIOP (Stockholm) working classification of renal tumours of childhood. International Society of Paediatric Oncology. Med Pediatr Oncol 1996;26:145-6.

11. Breslow NE, Churchill G, Nesmith B, Thomas PR, et al. Clinicopathologic features and prognosis for Wilms' tumor patients with metastases at diagnosis. Cancer 1986;58:2501-11.

12. Weeks DA, Beckwith JB, Luckey DW. Relapse-associated variables in stage I favorable histology Wilms' tumor. A report of the National Wilms' Tumor Study. Cancer 1987;60:1204-12.

13. Breslow N, Sharples K, Beckwith JB, et al. Prognostic factors in nonmetastatic, favorable histology Wilms' tumor. Results of the Third National Wilms' Tumor Study. Cancer 1991;68:2345-53.
14. Aloi I, De Ioris MA, Madafferi S, Jenkner A, Inserra A. Microscopic thrombosis in intermediate risk nephroblastoma. Pediatr Blood Cancer 2007;49:408.

15. Kaplan EL, Meier P. Nonparametric estimation from incomplete observation. J Am Stat Assoc 1959;53:457-81.

16. Faria P, Beckwith JB, Mishra K, et al. Focal versus diffuse anaplasia in Wilms tumor - new definitions with prognostic significance: a report from the National Wilms Tumor Study Group. Am J Surg Pathol 1996;20:909-20.

17. Grundy PE, Breslow NE, Li S, et al. Loss of heterozygosity for chromosomes $1 p$ and $16 q$ is an adverse prognostic factor in favorable-histology Wilms tumor: a report from the National Wilms Tumor Study Group. J Clin Oncol 2005;23:7312-21.

18. Dome JS, Bockhold CA, Li SM, et al. High telomerase RNA expression level is an adverse prognostic factor for favorable-histology Wilms' tumor. J Clin Oncol 2006;23:9138-45.

19. Miller MA, Karacay B, Breslow NE, et al. Prognostic value of quantifying apoptosis factor expression in favourable histology wilms tumors. J Pediatr Hematol Oncol 2005;27:11-4.

20. Diomedi Camassei F, Arancia G, Cianfriglia M, et al. Nephroblastoma: multidrug resistance P-glycoprotein expression in tumor cells and intratumoral capillary endothelial cells. Am J Clin Pathol 2002;117:484-90. 